\title{
Uso de Anfibios como Indicadores del Éxito de la Restauración de Ecosistemas 1
}

Ken G. Rice, Frank J. Mazzotti, J. Hardin Waddle y Marianna D. Conill ${ }^{2}$

\section{Introducción}

Existen 18 especies de anfibios nativos en la Florida del sur. Los anfibios se encuentran prácticamente en todos los hábitats, desde bosques de pinos hasta pantanos de juncias y estuarios de mangle, y pueden volverse extremadamente abundantes localmente (Figuras 2 y 5). Los anfibios son depredadores de artrópodos y algunos vertebrados pequeños y son presa de muchos otros animales (Fig. 1). Estas interacciones tróficas y la gran abundancia de anfibios en la Florida del sur los hace una parte vital del ecosistema de los Everglades.

Científicos alrededor del mundo han documentado disminuciones en las poblaciones de anfibios en muchas regiones y tipos de hábitats (Alford y Richards 1999). No se ha demostrado alguna causa específica para estos disminuciones, pero se han sugerido distintos estresores como precipitaciones ácidas, contaminantes ambientales, la introducción de predadores éxoticos, agentes infecciosos, parásitos y los efectos de radiación ultravioleta. Por su susceptibilidad a estos y otros estresores, los anfibios son indicadores importantes de la salud del ecosistema (Welsh y Ollivier 1998).

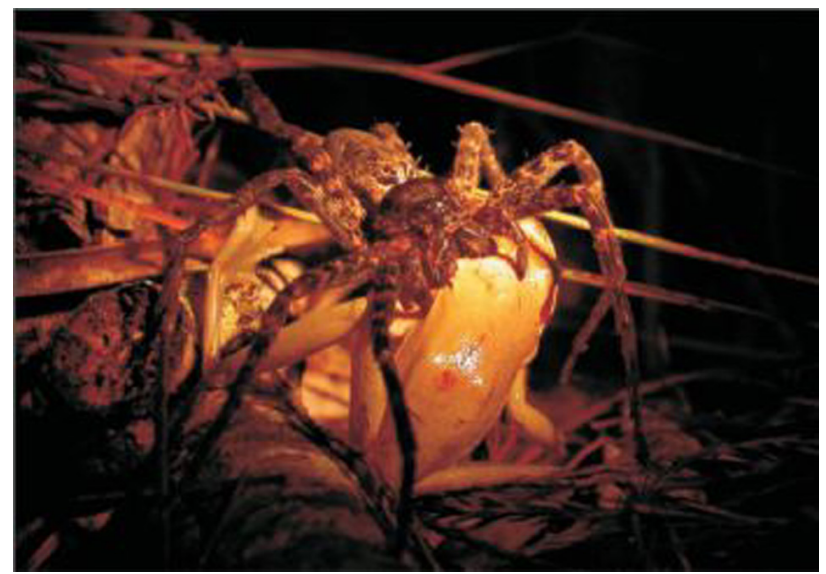

Figura 1. La araña de nombre común "Fishing Spider" de Okefenokee (Dolomedes okefinokensis) digiriendo una Rana Verde (Hyla cinerea) en una bóveda de ciprés en el Big Cypress National Preserve. Este es uno de los pocos casos documentados de artrópodos como predadores de ranas. Créditos Fotográficos: Brian Jeffery. Credits: Brian Jeffery, 2004

Actualmente, se está comenzando la restauración hidrológica en la Florida del sur bajo el

1. Este documento, CIR 1484S, es uno de una serie de publicaciones del Departamento de Wildlife Ecology and Conservation, Servicio de Extensión Cooperativa de la Florida, Instituto de Alimentos y Ciencias Agrícolas, Universidad de la Florida. (UF/IUFAS). Fecha de primera publicación: Noviembre 2006. Visite nuestro sitio web EDIS en 〈http://edis.ifas.ufl.edu〉.

2. Ken G. Rice, U.S. Geological Survey, 3205 College Ave, Davie, FL 33314, E-mail: ken_g_rice@ usgs.gov. Frank J. Mazzotti, University of Florida, 3205 College Ave, Davie, FL 33314, E-mail: fjma@ufl.edu. J. Hardin Waddle, Florida Cooperative Fish \& Wildlife Research Unit P.O. Box 110485 University of Florida Gainesville, FL 32611, E-mail: hardin_waddle@usgs.gov. Marianna Domínguez Conill, University of Florida, 3205 College Ave, Davie, FL 33314, E-mail: mconill@ufl.edu.

El Instituto de Alimentos y Ciencias Agrícolas es un empleador que opera bajo Acción Afirmativa y provee Oportunidades Igualitarias, autorizado a proveer investigación, información educativa y otros servicios, únicamente a los individuos e instituciones que operan sin discriminación alguna con relación al credo, color, religión, edad, incapacidad, sexo, orientación sexual, estado civil, nacionalidad, opinion política o afiliaciones. Para más información sobre como obtener otras publicaciones de extensión, comuníquese con la oficina de Servicio de Extensión de su condado. Servicio de Extensión de la Florida / Instituto de Alimentos y Ciencias Agrícolas / Universidad de la Florida / Larry Arrington, Decano. 
Plan Comprensivo de la Restauración de los Everglades (CERP, por sus siglas en inglés, www.evergladesplan.org), el cual incluye más de 68 proyectos que varían desde la remoción de canales hasta la creación de áreas de almacenamiento de agua. Los objetivos del plan incluyen el incremento de la extensión espacial de áreas naturales y el mejoramiento tanto de la calidad de las áreas naturales existentes como de la abundancia y diversidad de plantas y animales nativos. Un componente importante del plan es un proceso de evaluación adaptativa que será utilizado para medir el desempeño del plan y poder recomendar cambios como fueran necesarios. La comunidad de anfibios en los Everglades fue elegida como un grupo importante para este proceso de evaluación gracias a la sensibilidad que presentan a los cambios del ambiente.

Los anfibios pueden ser excelentes indicadores del éxito de la restauración de los Everglades, pero se requiere una clara comprensión de cómo los anfibios responderán a los cambios del ambiente. Actualmente, en un esfuerzo en colaboración, el U.S. Geological Survey (USGS) y University of Florida están dirigiendo un programa de investigación para el uso de anfibios como especies indicadoras de la restauración del ecosistema. Este programa consiste en proyectos de investigación coordinados que se llevan a cabo a lo largo de escalas tanto espaciales como temporales alrededor del ecosistema de los Everglades en el Everglades National Park, en el Big Cypress National Preserve y en los Water Conservation Areas. El objetivo de esta investigación es el desarrollo de herramientas necesarias para predecir y monitorear los cambios en las comunidades de anfibios como consecuencia de la restauración.

\section{Escala Local, Especie Individual}

Mientras los gerentes diseñan e implementan proyectos individuales del CERP, se deben evaluar los impactos sobre las especies indicadoras en áreas relativamente pequeñas. Los gerentes necesitarán de herramientas de predicción y métodos de monitoreo que puedan detectar cambios rápidamente y que se puedan aplicar a lo largo del sistema de los Everglades. A esta escala, los efectos de restauración sobre el tamaño poblacional, salud, comportamiento y reproducción de especies indicadoras individuales podrían ser utilizados para juzgar el éxito de restauración. La investigación a escala local consiste en el perfeccionamiento de métodos para estudiar y monitorear anfibios en el campo y en la utilización de estos métodos para hacerse preguntas apropiadas.

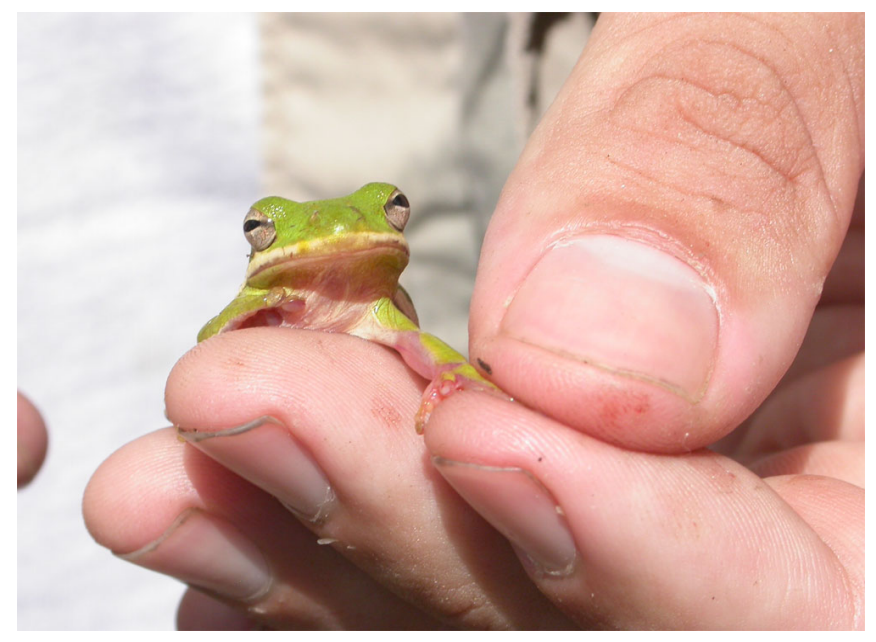

Figura 2. Rana Verde (Hyla cinerea) capturada en los pantanos del Big Cypress National Preserve. Créditos Fotográficos: Brian Jeffery. Credits: Brian Jeffery, 2003

La Rana Verde (Hyla cinerea) y la Rana Ardilla o Camaleónica (Hyla squirella) fueron las especies de anfibios más comúnmente observadas durante nuestros inventarios recientes del Everglades National Park y del Big Cypress National Preserve. Estas especies fueron encontradas en todos los hábitats que estudiamos (Fig. 3). En muchos casos se observó la presencia de ambas especies juntas, pero la densidad relativa de cada especie difiere dependiendo de las condiciones locales del hábitat. La salud y densidad de estas especies son indicadores tanto de la salud de los niveles tróficos inferiores de los que éstas se alimentan así como de sus predadores. Estamos utilizando técnicas de captura, marcaje y recaptura para examinar los patrones de movimientos y de supervivencia de ambas especies simultáneamente en el Big Cypress National Preserve. Estamos utilizando modelos multiestado para determinar la probabilidad de que una Rana Verde sobreviva y se mueva de un hábitat a otro con diferentes niveles de agua. Estos modelos toman en cuenta profundidades de agua y temperaturas, tanto actuales como del pasado, y nos permiten calcular una probabilidad de dispersión para cada especie 
bajo estas condiciones. Armados con esta información, estamos desarrollando un modelo de predicción para las poblaciones de ranas verdes bajo distintos escenarios hidrológicos. Los objetivos son desarrollar más aún nuestros métodos como una herramienta de modelaje y monitoreo a lo largo de todos los hábitats de los Everglades.

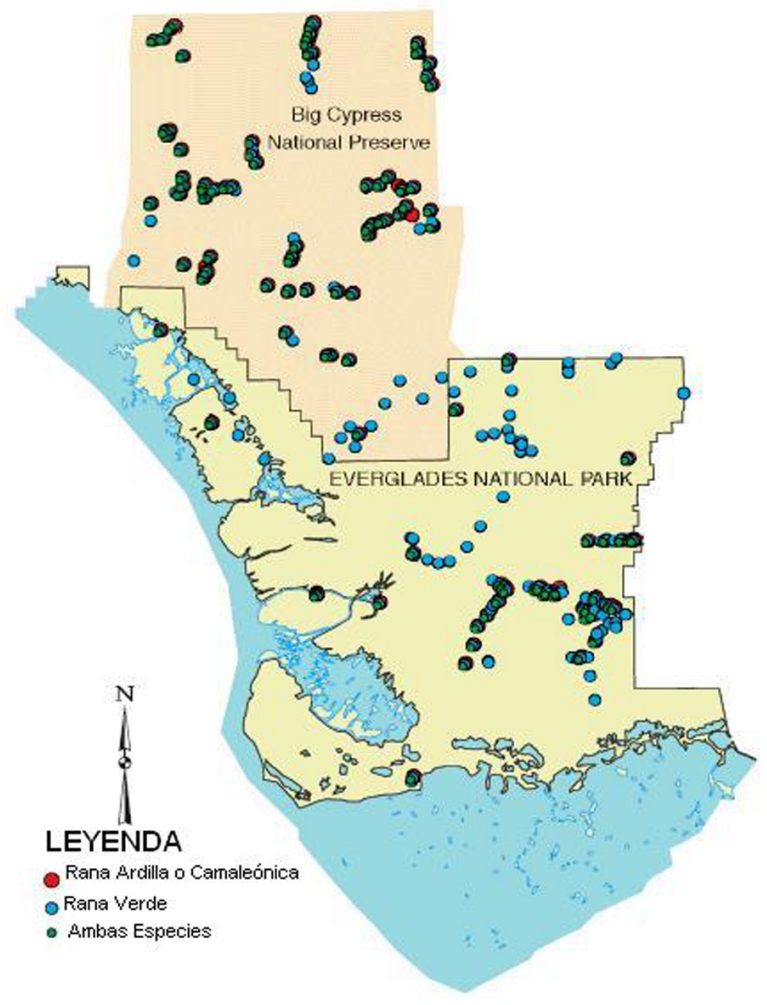

Figura 3. Localización de la Rana Verde (Hyla cinerea) y la Rana Ardilla o Camaleónica (Hyla squirella) dentro del Everglades National Park y del Big Cypress National Preserve. Credits: Brian Jeffery, 2004

\section{Escala Regional, Múltiples Especies}

Los efectos de los proyectos de restauración pueden ser medidos en una escala regional (tales como tipo de hábitat o unidad de manejo). En algunos casos, los efectos pueden ser difíciles de medir y puede que se requiera del uso colectivo de varias especies distintas para medir el éxito de restauración.

Otro gran impacto sobre los anfibios en los Everglades puede ser la introducción de especies invasoras. Estamos utilizando métodos similares de captura, marcaje y recaptura para determinar mejor el efecto de la Rana Platanera (Osteopilus septentrionalis) sobre las poblaciones de ranas nativas. Se ha mostrado que las Ranas Plataneras son predadores de al menos seis especies de ranas y sapos nativos (Meshaka 2001) y de al menos una especie de culebra nativa (Maskell y otros, 2003), y se sabe que estas Ranas Plataneras se han establecido en las áreas naturales dentro del Everglades National Park. Monitoreamos poblaciones de especies tanto nativas como introducidas utilizando datos de captura en refugios artificiales (Fig. 4). Después de un año de monitoreo, todas las Ranas Plataneras capturadas fueron removidas del área, y la recuperación de las ranas nativas fue monitoreada. Los resultados de este estudio indicaron que las Ranas Plataneras pueden causar reducciones severas en la abundancia y supervivencia de las ranas nativas en áreas donde la abundancia de la rana platanera es alta. Este planteamiento también podría ser aplicado a otros estresores de las poblaciones de anfibios, incluyendo la alteración del ciclo hidrológico.

\section{Escala de Paisaje, Comunidades}

Los gerentes del proyecto CERP necesitarán información acerca del éxito de restauración a lo largo del sistema total de los Everglades. Esto requerirá no sólo de métodos que sean aplicables a todos los hábitats y áreas sino también a muchas especies o comunidades.

Los anfibios están presentes en todos los hábitats y bajo todos los regímenes hidrológicos en los Everglades. Las tasas de ocupación- la proporción de un área o hábitat ocupada (PAO) por una especie en particular- y presencia difieren considerablemente a lo largo de esos gradientes. Estas diferencias se deben a los hidropatrones, vegetación y otros factores ambientales. La combinación de la composición de especies y la proporción de cada hábitat ocupado en un momento determinado forma comunidades únicas definidas por esos factores ambientales. Por lo tanto, si estas comunidades pueden ser definidas y medidas confiablemente, el éxito de restauración de los Everglades puede ser evaluado, los objetivos de restauración pueden ser establecidos, y las alternativas de restauración pueden ser comparadas. 


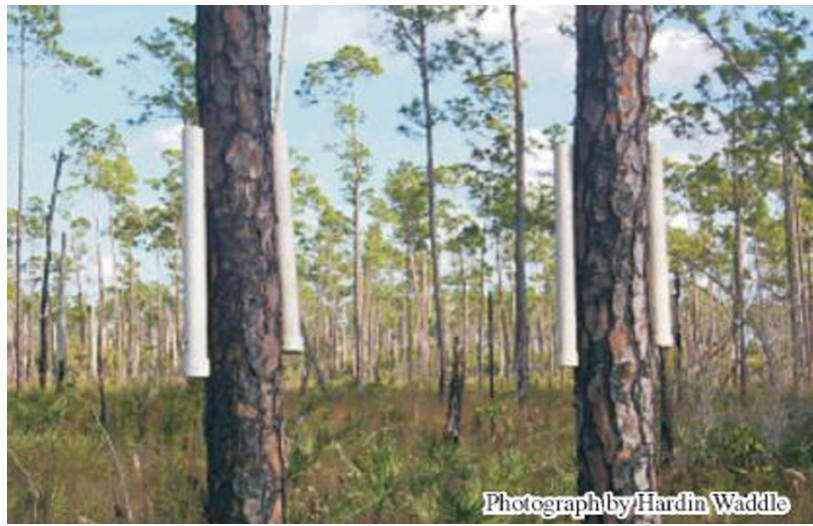

Figura 4. Tubos de cloruro de polivinil (PVC) utilizados como refugio artificial para capturar ranas verdes en un bosque de pinos en el Everglades National Park. Créditos Fotográficos: Hardin Waddle. Credits: Hardin Waddle, 2002

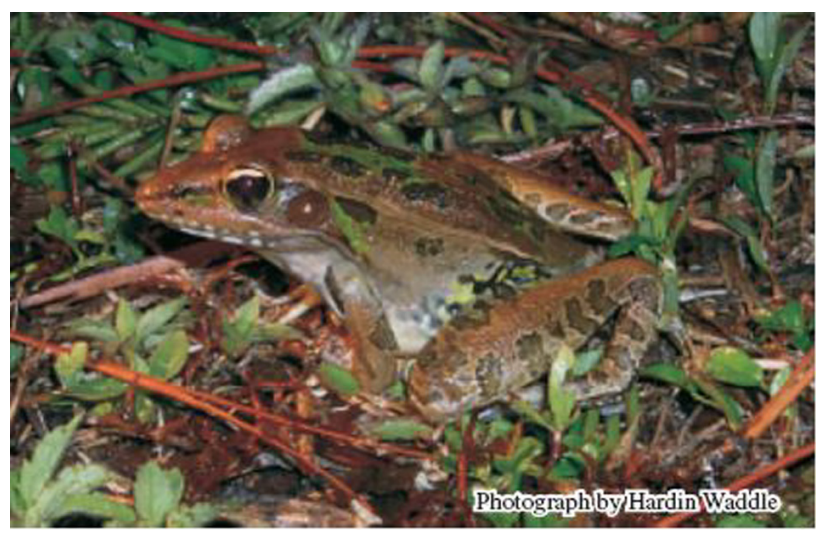

Figure 5. La Rana Leoparda del Sur (Rana sphenocephala) una rana relativamente grande que frecuenta los bordes someros de los humedales herbosos y los bosques de ciprés inundados en la Florida del Sur. Esta es una especie que probablemente refleje cambios en los espacios que ocupa durante la restauración de los Everglades. Créditos Fotográficos: Hardin Waddle. Credits: Hardin Waddle, 2002

En el Everglades National Park y los Water Conservation Areas adyacentes, hemos comenzado un estudio a gran escala para determinar la PAO por cada especie de anfibio a lo largo de hábitats definidos por hidropatrón (el número de días de inundación cada año). El método PAO estima la abundancia de sitios en los cuales cada especie ocurre basándose en los resultados de captura de varias visitas a cada sitio (MacKenzie y otros, 2002). Este método toma en cuenta que algunas especies son más difíciles de detectar (sabiendo que están presentes) que otras. Este muestreo se lleva a cabo a lo largo de un gradiente hidrológico desde pantanos, que presentan largos hidroperíodos, hasta áreas rocosas denominadas "rocky glades" del este del Everglades National Park, que presentan hidroperíodos extremadamente cortos. Los resultados de esta investigación serán perfiles de comunidad que relacionan la PAO de varias especies de anfibios clave con la comunidad ecológica a lo largo de este gradiente. Esta información luego puede ser utilizada por los gerentes para hacer predicciones y monitorear el éxito de CERP (Fig. 6).

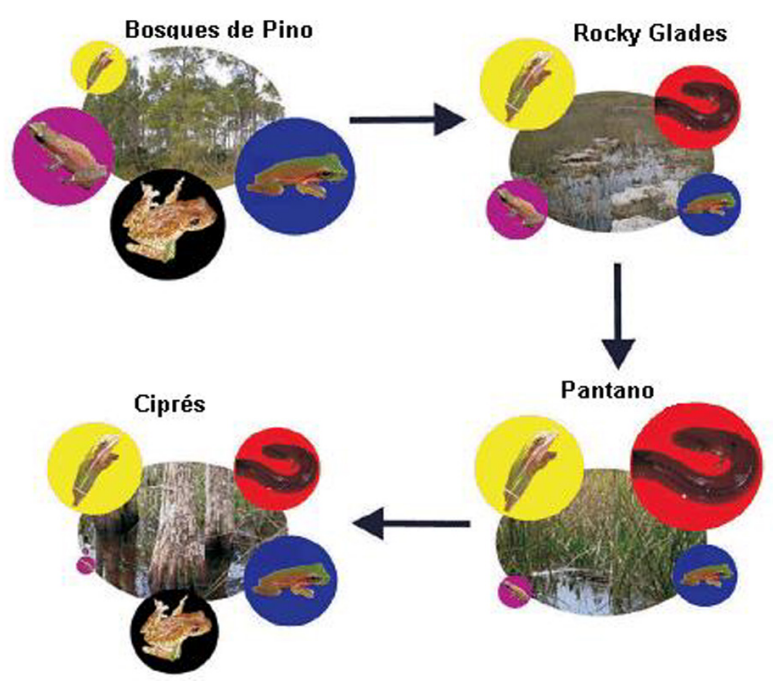

Figura 6. Diagrama mostrando cómo la proporción de área ocupada (PAO) por varias especies de anfibios puede diferir en cuatro tipos de hábitat. Las flechas indican la dirección del aumento del hidroperíodo, desde muy pocos días en hábitat de montes de pinos, hasta casi inundación permanente en hábitat de ciprés. El tamaño del círculo alrededor de cada anfibio representa la PAO para esa especie en ese hábitat. Las Ranitas de Grama o "Little Grass Frogs" por su nombre común en inglés (círculo rosado) son relativamente abundantes en los montes de pinos, pero diminuyen en términos de PAO en hábitats más húmedos. Las Sirenas Mayores (círculo rojo) sólo se encuentran en hábitats más húmedos, pero alcanzan su mayor PAO en hábitats de pantano abierto. Credits: Hardin Waddle, 2004

También estamos utilizando esta aplicación del modelo de modelaje para determinar el impacto que generan los vehículos que se trasladan fuera de las vías (ORV, por sus siglas en inglés) sobre los anfibios en el Big Cypress National Preserve (Fig. 7). Primero desarrollamos un índice del uso de ORV utilizando sistemas de información geográfica (GIS, por sus siglas en inglés) (Fig. 8). Luego, probamos el modelo PAO usando técnicas de selección simulada para determinar si el uso de ORV 
afectaba la distribución de anfibios dentro de la Reserva. Los resultados indicaron que algunas especies parecen estar fuertemente asociadas de manera negativa al uso de ORV, algunas parecen estar fuertemente asociadas positivamente, y muchas parecen no verse afectadas por el uso de ORV. Este es un ejemplo de una aplicación de este modelo para probar si los procesos a escala de paisaje afectan la distribución de especies de anfibios en la Florida del sur. Otros factores, tales como hábitat e hidroperíodos, también pueden ser incluidos en el modelo para determinar la serie de factores que mejor explican la distribución de las especies.

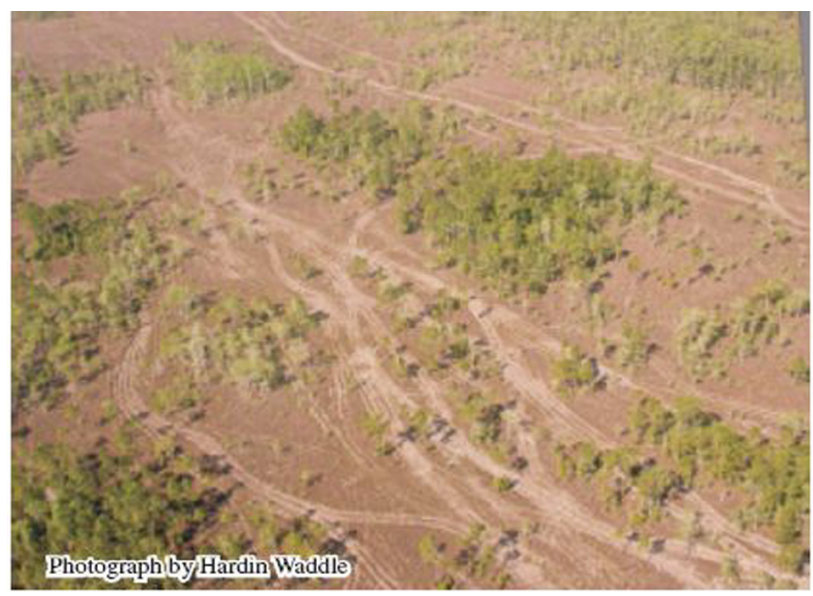

Figura 7. Rastros dejados por los vehículos que se trasladan fuera de las vías (ORV) en los llanos del Big Cypress National Preserve. Créditos Fotográficos: Hardin Waddle. Credits: Hardin Waddle, 2004

\section{Anfibios y la Restauración de los Everglades}

Durante la restauración de los Everglades, los gerentes requerirán de predicciones acerca de cómo los cambios propuestos podrían afectar a las especies indicadoras y de datos de monitoreo que aseguren el éxito de restauración. Se necesitará que los métodos abarquen desde escalas espaciales pequeñas hasta paisajes y desde especies individuales hasta comunidades. Mediante la descripción de las distribuciones y abundancias de anfibios actuales a lo largo de los Everglades y el mejor entendimiento de las relaciones entre hidrología y poblaciones de anfibios, podremos hacer predicciones acerca de los efectos de la restauración de los Everglades. El monitoreo de anfibios utilizando las técnicas que estamos

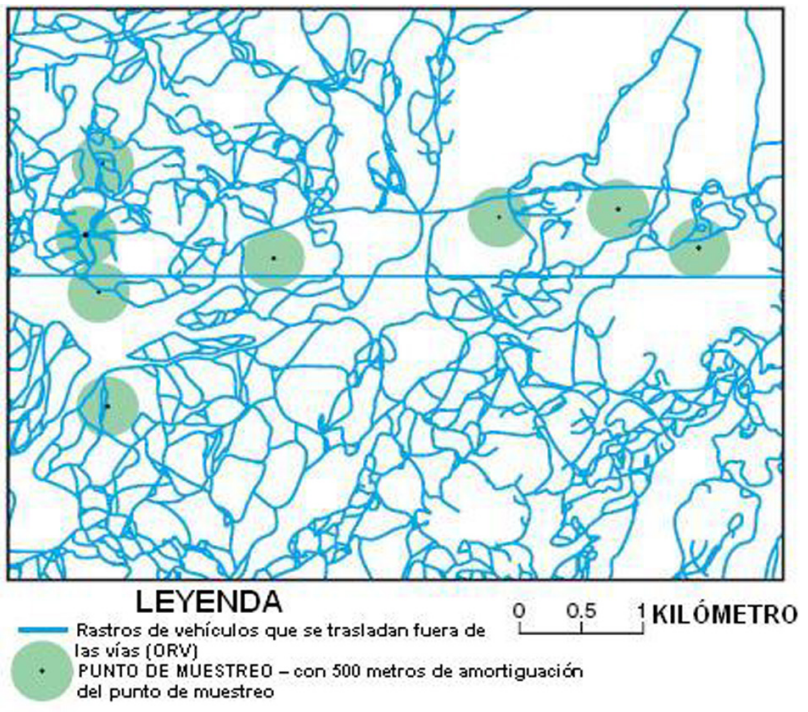

Figura 8. Ocho puntos de muestreo rodeados de 500 metros de zona amortiguadora. Las líneas azules representan los rastros dejados por vehículos que se trasladan fuera de las vías (ORV), y el largo de los segmentos de rastros dentro de los círculos verdes son la base para el índice de ORV. Credits: Hardin Waddle 2004; Adaptado de Welch, et. al 1998

desarrollando permitirá poner a prueba las predicciones y medidas del éxito de restauración. Tanto el USGS Greater Everglades Priority Ecosystem Science (PES) como el programa U.S. National Park Service CESI han sustentado esta investigación.

\section{Referencias Bibliográficas}

Alford, R.A. y Richards, S.J. 1999. Global amphibian declines: A problem in applied ecology. Annual Review of Ecology and Systematics, 30, 133-165.

MacKenzie, D.I., Nichols, J.D., Lachman, G.B., y otros. 2002. Estimating site occupancy rates when detection probabilities are less than one. Ecology, 83, 2248-2256.

Maskell, A.J., Waddle, J.H., y Rice, K.G. 2003. Osteopilus septentrionalis: Diet. Herpetological Review, 34, 137.

Meshaka, W.E. 2001. The Cuban Treefrog in Florida. Gainesville, University of Florida Press. 
Welch, R., M. Madden, and R. F. Doren. 1999.

Mapping the Everglades. Photogrammetric

Engineering and Remote Sensing, 65, 163-170.

Welsh, H.H.J., y Ollivier, L.M. 1998. Stream

amphibians as indicators of ecosystem stress: A case

study from California's redwoods. Ecological

Applications, 8, 1118-1132. 